\title{
Current state of prognostication, therapy and prospective innovations for Barrett's-related esophageal adenocarcinoma: a literature review
}

\author{
Sumeet K. Mittal ${ }^{1}$, Joe Abdo ${ }^{2}$, Malika P. Adrien ${ }^{3}$, Binyam A. Bayu ${ }^{3}$, Jay R. Kline ${ }^{3}$, Molly M. Sullivan ${ }^{3}$, \\ Devendra K. Agrawal ${ }^{4}$
}

${ }^{1}$ Norton Thoracic Institute, St. Joseph's Hospital and Medical Center, Dignity Health, Phoenix, AZ, USA; ${ }^{2}$ Stella Diagnostics, Inc., Salt Lake City, UT, USA; ${ }^{3}$ Department of Biochemistry, Georgetown University Medical Center, Washington, DC, USA; ${ }^{4}$ Department of Translational Research, Western University of Health Sciences, Pomona, CA, USA

Contributions: (I) Conception and design: SK Mittal, DK Agrawal; (II) Administrative support: MP Adrien, BA Bayu, JR Kline, MM Sullivan; (III) Provision of study materials or patients: MP Adrien, BA Bayu, JR Kline, MM Sullivan, J Abdo; (IV) Collection and assembly of data: MP Adrien, BA Bayu, JR Kline, MM Sullivan; (V) Data analysis and interpretation: SK Mittal, DK Agrawal, J Abdo; (VI) Manuscript writing: All authors; (VII) Final approval of manuscript: All authors.

Correspondence to: Sumeet K. Mittal, MD, FACS. Professor of Surgery, Norton Thoracic Institute, St. Joseph's Hospital and Medical Center, Dignity Health, 500 W Thomas Rd Suite 500, Phoenix, AZ 85013, USA. Email: Sumeet.Mittal@dignityhealth.org.

Objective: Barrett's esophagus (BE) is the only known precursor to esophageal adenocarcinoma (EAC), which has one of the lowest 5-year survival rates in oncology. The reasons for poor survival are twofold: the large majority of diagnoses are in advanced stages $(\sim 0 \%)$ and limited treatment options, with a deficit of biology-guided therapies. As a rapidly growing public health concern with poor prognosis, research into the molecular progression for BE and novel therapeutics for EAC currently has high clinical utility. Review of the literature reveals that innovative analysis of metaplastic progression from BE to EAC at a molecular level can shed light on the underlying transformative probabilities of BE into malignant pathologies and may impact current of future therapeutic modalities for management of these diseases.

Background: EAC is the fastest increasing cancer in the United States with a $600 \%$ increase over the past 25 years. This cancer arises from dysplastic tissue of BE, a complication of gastroesophageal reflux disease (GERD). Chronic acid and bile reflux in the distal esophagus initiates a metaplastic conversion of normal squamous epithelium to premalignant intestinalized columnar epithelium. Patients with BE have a 125-fold higher risk of cancer compared to the general population.

Methods: We critically reviewed the current status of BE monitoring, and subsequent therapeutic strategies being used in patients who have progressed to cancer. Also, new diagnostic tools and therapeutic candidates for BE-related EAC are discussed. Highly-targeted searches of databases containing recent original peerreviewed papers were utilized for this review.

Conclusions: Novel and well-described biomarkers analyzed in the patient's diseased tissue will provide for more powerful diagnostics, but also possess the potential to develop strategies for personalized management and identify targets for intervention to either cease disease progression or treat BE and/or EAC. Since millions of Americans develop BE without progressing to cancer, there is a critical need to identify the small percentage of Barrett's patients who possess hallmarks of disease progression or carcinogenesis with novel screening techniques. Incorporation of such tools into standard screening protocols for BE surveillance and/or therapy would be critical to detect malignant transformations before clinically obvious cancer ever develops. 


\begin{abstract}
Keywords: Barrett's esophagus (BE); esophageal adenocarcinoma (EAC); precision oncology; targeted therapy;
\end{abstract} early cancer detection

Submitted Mar 03, 2021. Accepted for publication Jun 30, 2021.

doi: 10.21037/jgo-21-117

View this article at: https://dx.doi.org/10.21037/jgo-21-117

\section{Introduction}

Esophageal cancer is the eighth-most common cancer (1). About $83 \%$ of esophageal adenocarcinoma (EAC) patients do not survive beyond five years (2). Gastroesophageal reflux disease (GERD) is the most common diagnosis and EAC has the greatest increase in incidence in the western world and the link between the two is Barrett's Esophagus (BE) - the only known precursor to EAC. Individuals with $\mathrm{BE}$ have a 125 -fold increased risk of developing esophageal cancer compared to the general population (3). BE most commonly arises from GERD, as up to $15 \%$ of GERD patients develop BE (4). It is estimated that a quarter of the US adult population suffers from GERD (5). Coinciding with increasing GERD and BE prevalence, EAC presents the highest increase in incidence rate among all cancer types $(2,6)$. Notably, EAC is the deadliest cancer by incidence, annually (Figure 1).

Due to this link between $\mathrm{BE}$ and $\mathrm{EAC}, \mathrm{BE}$ patients are monitored to identify EAC development. The increased incidence of EAC and severe lethality warrant routine screening and surveillance of BE. Diagnosis occurs through a combination of endoscopic and histological analyses (7). This leads to BE classification of one of the following: nondysplastic, indeterminate-grade dysplasia, low-grade dysplasia (LGD), and high-grade dysplasia (HGD) (8). The cascade of increasing dysplasia ultimately leads to malignant degeneration (8). This classification is used to guide BE management strategies, per the American Society for Gastrointestinal Endoscopy (ASGE) (7). More severe dysplasia warrants more frequent endoscopic surveillance follow-ups and increasingly proactive intervention (7).

However, no randomized, controlled trials have shown endoscopic BE screening to improve patient survival rates (7). At best, only $15 \%$ of EAC cases are identified during routine surveillance (9) and these patients have better clinical outcomes compared to those found incidentally. For patients at lower risk, BE monitoring and surveillance lack cost-effectiveness $(10,11)$. Yet, patients considered to be low-risk for disease progression can develop EAC during routine surveillance (10). Due to the histological and subjective nature of BE monitoring, interobserver agreement among physicians can vary greatly, demonstrating the inefficiencies of current methods (11). A more robust system for grading risk for malignant progression is warranted. Studies investigating the utility of molecular and genetic biomarkers have shown potential to mitigate the difficulties in risk stratification for EAC pathogenesis to better inform interventional strategies (11).

Current treatment of EAC has several clinical limitations. EAC has one of the highest mortality rates in cancer, and $\mathrm{BE}$ has a 10 -year mortality rate that is similar or higher than other neoplasms (Figure 2). More than half of patients diagnosed with EAC have already progressed to late-stage carcinogenesis at the time of diagnosis, which coincides with a five-year survival rate below $5 \%$ (12). Studies have reported that chemotherapy has no effect on survival rates of EAC patients with distant metastases (12). Median survival rate is the same for patients undergoing various chemotherapy regimens compared to patients who received no therapy in stage IV EAC cohorts (12). The only FDAapproved targeted therapy for EAC is trastuzumab that targets HER2, which is overexpressed in only $7-22 \%$ of EAC tumors (13). Addition of trastuzumab to chemotherapy increases survival by three months (14). Several biomarkers known to play roles in chemotherapy resistance have been found to be overexpressed in EAC tissue (15). Ongoing research is investigating targeted therapies within the pathways of epidermal growth factor receptor (EGFR) and vascular endothelial growth factor (VEGF) (16). However, currently molecular diagnostics do not play a role in EAC therapeutic decision-making, and targeted therapies rely on this quantitative diagnostic guidance (16).

We present the following article in accordance with the Narrative Review reporting checklist (available at https:// dx.doi.org/10.21037/jgo-21-117).

\section{Methods}

Relevant literature was collected through the use of private 


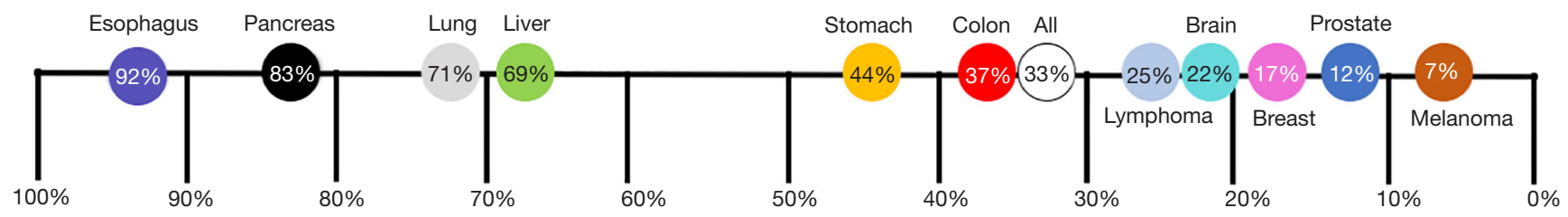

Figure 1 Cancer deaths relative to incidence annually (percentage). Esophageal adenocarcinoma is the deadliest cancer in terms of deaths relative to annual incidence. Strikingly, the rate of incidence of EAC is rising the faster than any cancer in the US, which could lead to major clinical implications in the future. EAC possess a higher deaths per incidence annually rate than some of the more publicized indications like pancreatic, lung, brain, breast, liver and colon cancer. EAC, esophageal adenocarcinoma.

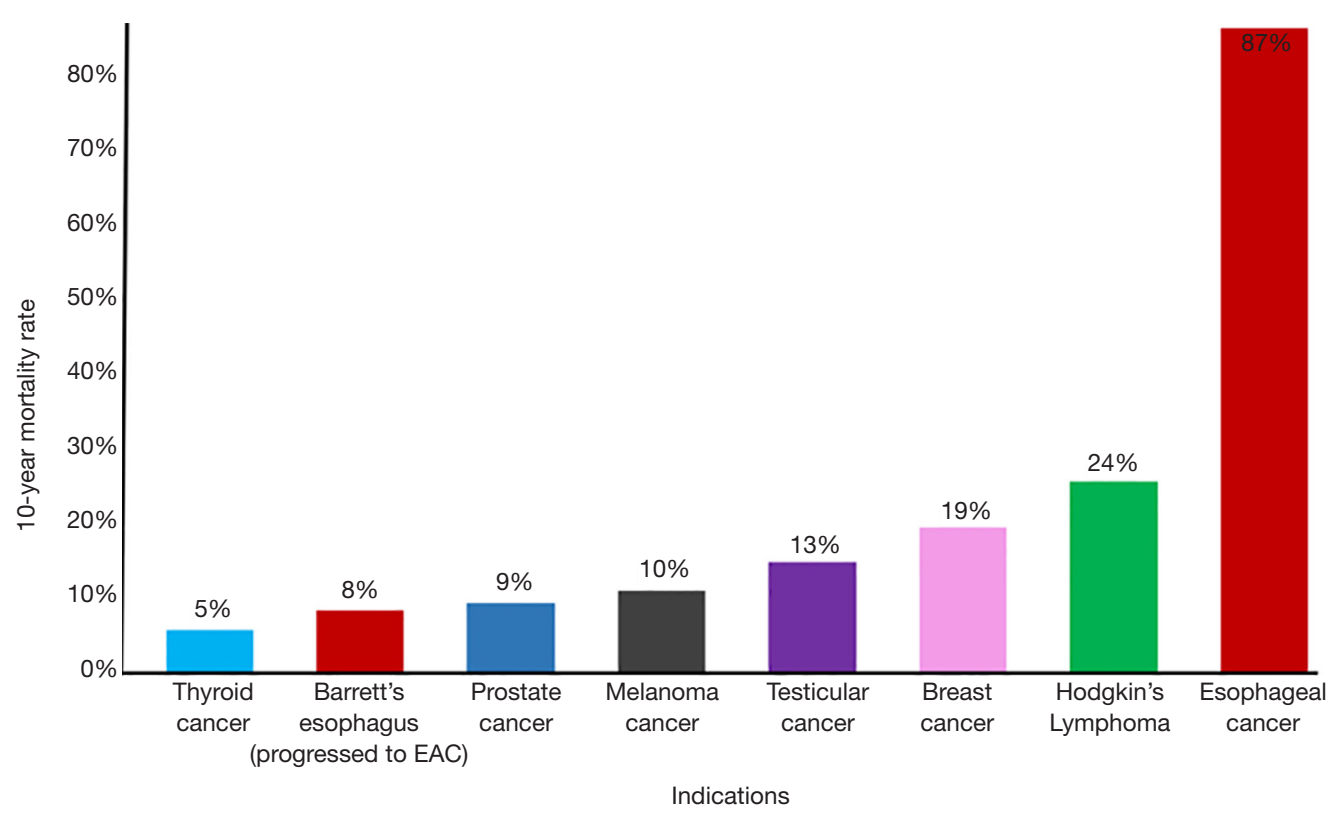

Figure 2 A 10-year mortality rate (Barrett's esophagus metaplasia vs. cancer). The 10-year mortality rate for Barrett's esophagus (non-cancerous disease) is higher or within the range of some cancers in the United States. Although indications like GERD and Barrett's esophagus are not considered very serious, it can be just as deadly as some of the well-described indications in oncology. Esophageal adenocarcinoma, which is linked to Barrett's esophagus, has one of the highest mortality rates in oncology. GERD, gastroesophageal reflux disease.

and governmental databases, hand searches, and high impact texts in order to amalgamate findings applicable to the current state of the EAC disease space. Information used to write this paper was collected from PubMed searches inspecting papers' pertinence to our objectives from peerreviewed manuscripts published in 2002 through 2020. The key words we used for our searches were: esophageal adenocarcinoma, targeted therapy, diagnostics, Barrett's esophagus, meta-analysis, gastro-esophageal reflux disease (GERD), standard of care, endoscopy, chemotherapy, genomics, proteomics and transcriptomics. Google Scholar searches from 2015 through 2020 utilizing the same key words was also conducted to elucidate the newest innovations in this field. We also utilized hand searches of the references of retrieved literature from the Georgetown University School of Medicine library searching for texts on relevant literature reviews. We also conducted discussions with experts in the fields of clinical oncology, gastroenterology, molecular diagnostics, and surgical oncology. The personal experience of the domain experts participating in the authoring of this review (i.e., Drs. Mittal, Abdo and Agrawal) were also incorporated into this manuscript.

\section{GERD}

It is important to recognize that there are distinct precursors 


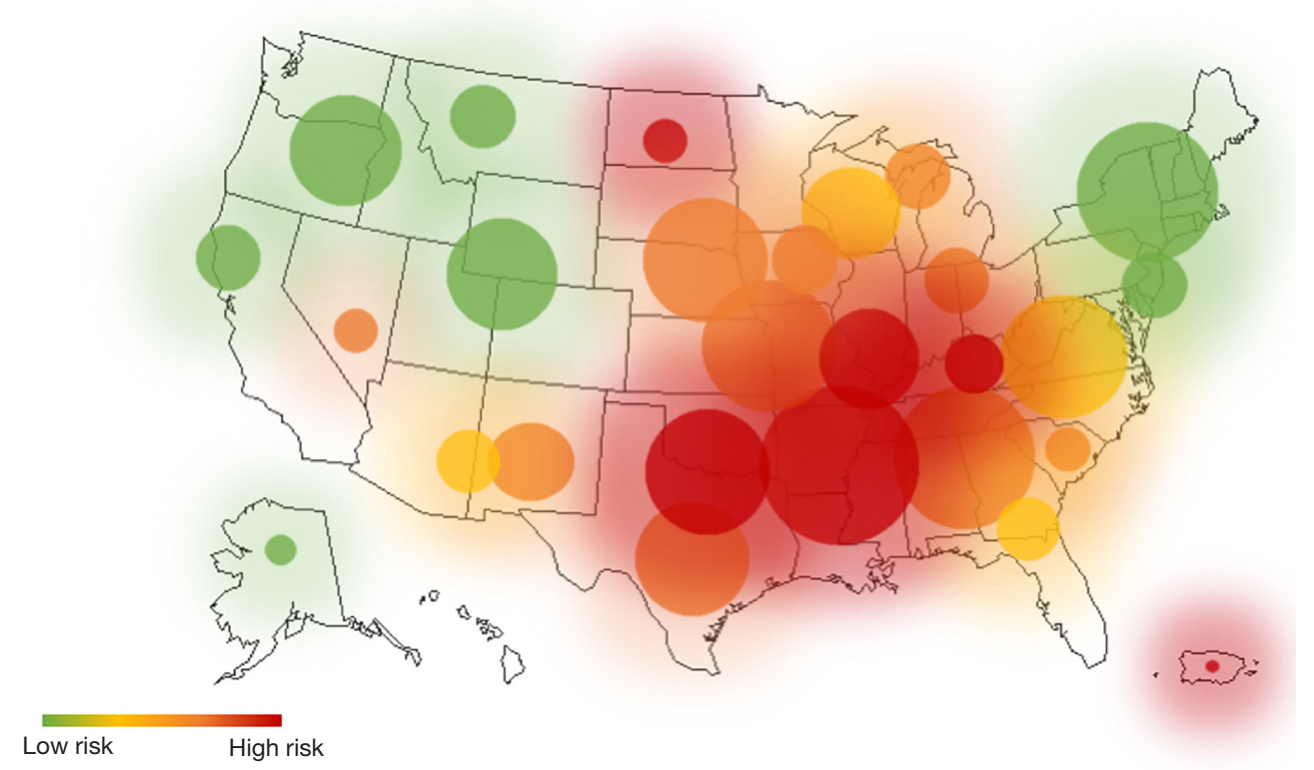

Figure 3 Prevalence of Barrett's esophagus in the US [2020]. A heatmap of the prevalence of BE by region within the US. The highest rates are found in the South, Southeast, and the Midwest. Some of the lowest rates of BE are seen on the West Coast and in the Northeast and New England. US, United States; BE, Barrett's esophagus.

before EAC develops. Currently, BE is the only known precursor to EAC. The prevalence of $\mathrm{BE}$ is increasing sharply along with EAC and a heat map of the United States shows that these indications are more prevalent in some areas of the country, namely the South and Midwest (Figure 3). GERD is the only known precursor to BE. GERD has a prevalence of $18-28 \%$ in the United States population (5). Patients with frequent GERD symptoms have a 3-5 times higher risk of developing EAC; while patients with severe and long-standing GERD symptoms have a 43 times more likely chance $(4,11)$. This highlights the importance of GERD monitoring and treatment. Despite its significant connection to BE and EAC, GERD is not routinely screened for disease progression. EsoGuard (Lucid Diagnostics, Inc.), is an Esophageal DNA Test offered as a Laboratory Developed Test that may be used to detect $\mathrm{BE}$, both non-dysplastic, as well as dysplastic. This test assays tissue for the presence of both methylated CCNA1 and Vimentin and offers a binary result based on a proprietary algorithm; two epigenetic biomarkers that have been demonstrated to be correlated at high sensitivity and specificity with BE (17). Methylated DNA biomarkers are attractive because these assays are automatable, inexpensive, and not subject to interobserver disagreement when inspecting disease hallmarks via histopathology.

Common treatment options for GERD include over the counter antacids and proton pump inhibitors (PPIs). While this may help treat GERD and alleviate symptoms, patients on PPIs can still exhibit pathologic esophageal acid exposure, and subsequently disease progression (18). It is this gastric acid exposure that leads to premalignant clinical manifestations such as BE and potentially a malignant EAC prognosis. This shows the need for enhancements in the standard of care for GERD screening, as a missed diagnosis could potentially be detrimental for the patient down the road. By using improved therapies and augmented molecular screening for GERD, there is potential of possibly lowering the number of cases that progress to EAC, by identifying BE patients at the point of care, or at the very least identify EAC patients when the disease is still in a non-advanced and curable state. The current clinical screening approach for GERD patients is not molecular-based and the ability to identify non-visible $\mathrm{BE}$ or short-segment $\mathrm{BE}$ can better define the cohorts of patients in need of distal esophageal screening and potentially candidates of therapeutic options. 


\section{Pathophysiology of malignant transformation of normal esophageal epithelium}

\section{Epidemiology}

Although BE is the primary risk factor for onset of EAC, the progression rate is estimated to be only less than one person per one-hundred (19). Thus, it has been suggested that further risk factors and predictors of progression be identified and monitored to bolster the identification of patients at risk of malignant transformation (20). Older age, male gender, and overweight status [body mass index (BMI) range, 25-29.9] are independent factors increasing the risk of $\mathrm{BE}$ progression to EAC, while PPIs and statins show protective effects against carcinogenesis (21-23). Dysplasia currently presents as the best predictor of malignant progression (24). However, this presents challenges in cases of indefinite dysplasia $(24,25)$. Recent progress in biomarker identification has highlighted potential for improvements in risk stratification $(24,26,27)$. However, biomarkers are not included in the guidelines set forth by GI societies (24). Nonetheless, risk stratification of patients into low-risk and high-risk groups for esophageal cancer provides clinical utility, and significant findings in recent years (e.g. age, sex, BMI, dysplasia) have promoted the adoption of this epidemiology-based risk stratification $(20,25)$.

\section{Changes in expression patterns and bistopathology}

There are several theories for the histopathological changes observed in the conversion of normal squamous esophageal epithelium to the metaplastic columnar cell structure observed in BE tissue (28). Several immunohistochemical markers have been identified such as CDX2, villin, MUC2 , but their consistent presence in BE cells is debated. One theory centers around the origination of $\mathrm{BE}$ cells in the stomach where CDX2 promotes intestinal differentiation upon gastric gland encounter with acid or bile (29). Other recent theories have focused on native esophageal or esophageal submucosal gland cells based on hypotheses of circulating stem cell recruitment, reactivation of dormant p63-/KRT7+ residual embryonic cells, or differentiation of p63+KRT5+KRT\& + transitional basal progenitors, noting several markers of interest including KRT8, SPINK4, ITLN1, LEFTY1, OLFM4 (30,31). Downregulation of p63 and SOX2, as well as upregulation of SOX9, CDX2 and FOXA2 have been shown to alter normal esophageal tissue into a morphology similar to columnar epithelium but did not result in the presence of goblet cells in $\mathrm{BE}$ metaplasia (32). BE cells have also been noted to have a proliferative advantage compared to squamous mucosal cells in an intermittently acidic environment (32).

\section{Changes in microenvironment}

The BE microenvironment resembles that of duodenal tissue, with a recent study citing the increased expression of RALDH2 and FOXP3 to potentially indicate an antiinflammatory, gut like microenvironment $(33,34)$. These findings has further been linked to unfavorable survival in EAC (35). Other studies have noted increased gramnegative bacteria and alpha and beta diversity in the $\mathrm{BE}$ microbiome when compared to normal esophageal mucosa (36-38).

\section{Tissue retrieval techniques}

\section{Routine endoscopies}

Esophagogastroduodenoscopy or "upper endoscopy" and the procurement of tissue samples are essential in the diagnosis and treatment for various diseases within the digestive system. If the endoscopic and subsequent histological examination of the distal esophagus reveals intestinal dysplasia/metaplasia, then patients are then officially diagnosed with $\mathrm{BE}$ and will be subject to routine following-up and screening to assess if the disease is stable, progressing or turning cancerous (39). BE presents on a visually inspected endoscopy characterized as a salmon-pink colored extension of mucosa that grows into the esophagus above the gastroesophageal junction (GEJ) (40). To screen and surveil, four quadrant biopsies are taken along every $2 \mathrm{~cm}$ of the BE-type mucosa (41). Although this approach samples for a small fraction of affected lining, this allows for the opportunity to recognize dysplasia. BE is traditionally termed as long segment if the extensions are $3 \mathrm{~cm}$ or more in length, short segment when less than $3 \mathrm{~cm}$, and ultra-short segment of less than $1 \mathrm{~cm}(42)$. The location of the biopsy is important, ultra-short $\mathrm{BE}$ is difficult to differentiate from irregular GEJ and is presumed to be significantly less at risk to develop cancer than traditional BE (43). During carcinogenesis, the tissue will develop morphologic changes related to unregulated cell growth that is recognized as dysplasia. However, active inflammation of the esophagus makes it difficult to discern if dysplasia is present or assess if other reparative changes are occurring (44).

The spectrum of change is subdivided into four clinically 
significant groups: negative for dysplasia, indeterminate for dysplasia, LGD and HGD to signify progressively increasing risk of progression to EAC. The degree of dysplasia is determined by evaluating the cytology, architecture, and degree of surface maturation and interpreting these findings in conjunction with the amount of surrounding inflammation. If the initial diagnostic biopsy of $\mathrm{BE}$ is negative for dysplasia, a repeat endoscopic exam is recommended within a year (44). If the second scope is also negative for dysplasia a follow up between 3 to 5 years is recommended. If low grade dysplasia is identified on initial presentation, a repeat exam is recommended within 6 months to check for higher grade dysplasia (42). For patients with LGD, the annual risk of progression to cancer is $0.7 \%$ per year, respectively (44). If HGD is diagnosed by the gastroenterologist and/or GI pathologists, then the recommendation is the patient should be considered for endoscopic ablative therapy after an additional confirmatory endoscopy (44). Biopsies are taken at $1 \mathrm{~cm}$ intervals, which suggests that a treatment of endoscopic mucosal resection should be done to mitigate further disease progression (42). More recently, proactive endoscopic ablation has been recommended for higher grades of dysplasia and T1a EAC in esophageal epithelium.

Endoscopies require sedation, specialized equipment/ units and clinical expertise. This inherently carries a high healthcare cost and potential risk to patients. Endoscopies are standard of care, but less invasive techniques to retrieve diseased pathological tissue in the distal esophagus are currently in development or are being commercialized.

\section{Non-invasive observation (serum markers)}

An early diagnosis of cancer could provide a much more hopeful and promising full recovery for the patient. The conventional methods of cancer screening are often invasive and expensive, sensitivity and specificity of these methods are also insufficient for diagnosis at an early stage. For this reason, researchers are attempting to increase sensitivity and specificity of early detection methods (45). Analyzing the cell free DNA (cfDNA) of a tumor is promising addition to non-invasive cancer detection. The cfDNA is a generic biomarker which is present in the serum or plasma in high concentrations. This identification of circulating cancer-related DNA molecules is a non-invasive technique for early diagnosis and prognosis in cancer patients with esophageal carcinoma (45). Levels of cfDNA in patients with esophageal cancer was shown to be significantly high and the cfDNA level returned to normal after complete resection of the tumor (46). The average circulating cfDNA concentrations from esophageal cancer patients are found to be significantly higher than those in healthy controls (47). The detection of the differences in cfDNA levels is a potential approach and less invasive tool for early detection of patients with carcinoma of the esophagus (45). However, this diagnostic approach is not presently useful for screening or prognostication of $\mathrm{BE}$ progression into cancer.

\section{Cytology}

Brush cytology has been examined in BE to reduce the need for repeated and continuous endoscopic biopsies (48). There are various cytology brushes available for tissue retrieval. The designs of these brushes included variable sizes and stiffness, wire guided, or non-wire guided, single or millilumen, and with or without a flexible guide tip (49). Cytology brushes are to be used tangentially with pinch biopsies which are helpful in the diagnoses of dysplasia, malignancies and infections (49). This technique allows larger areas of esophagus epithelium to be sampled (50). However, there is a tendency for dysplastic cells to be break free or slough off more readily than normal cells, so this technique could augment the sample collection for diseased esophageal tissues (50). A computer assisted brush biopsy technique (EndoCDx; CDx Technologies) has been utilized to aid in the diagnosis of BE. In this procedure, a stiff endoscopic brush device can sample deeper layers of the esophageal epithelium which in turn will provide further information about cell populations and structures. The samples are analyzed by a high-speed automated process and artificial intelligence software with a trained pathologist that can identify abnormal cells and glandular structures with features of BE or dysplasia (50). Although endoscopic cytological analysis has some clinical advantages compared to endoscopic biopsy analysis, it is still prone to sampling errors and high falsepositive rates for detection of dysplasia (50). There are several companies worldwide producing these products, paving the way for a less invasive approach for BE tissue collection.

\section{EsoCheck}

The EsoCheck (Lucid Diagnostics, Inc.) cell collection device is a $510(\mathrm{k})$ cleared non-invasive device that offers collection of distal esophageal mucosal cells to test for BE and EAC with a DNA biomarker test. During 
this brief office-based procedure, a patient swallows a vitamin pill sized capsule containing a small inflatable balloon attached to a thin catheter. As the catheter is withdrawn, the inflated balloon swabs the target area to collect a sample of cells. Deflation of the balloon prior to withdrawal serves to protect the sample from dilution or contamination. The collected tissue may then be assessed via a panel of methylated biomarkers to detect $\mathrm{BE}$ or EAC. Additional uses for EsoCheck beyond detection of BE/EAC are under study. One example is a study by Falk et al. titled "EsoCheck Compared to Biopsies and Brush Cytology During Endoscopy for Evaluation of Eosinophilic Esophagitis". This trial is a prospective cross-sectional pilot feasibility study of ten patients with suspected or established eosinophilic esophagitis for a clinically indicated upper endoscopy $(51,52)$.

\section{WATS3D ${ }^{\circledR}$ brush biopsy}

WATS3D transepithelial biopsy has been created to augment traditional biopsy with the intention of better detection of dysplasia and EAC. A study was performed to compare the variability of detection of $\mathrm{BE}$, degree of dysplasia and adenocarcinoma of WATS3D brush biopsy to traditional forceps biopsy (53). WATS transepithelial biopsy allows for a wide full-thickness biopsy to be obtained along with computer assisted 3D analysis in which 200 of the most suspect cells and cell clusters are analyzed (53). By taking a wider biopsy with WATS technology, the rate of detection of BE and EAC may be higher, and augment better detection rates than traditional specimen-retrieval approaches (53). A moderate agreement in the findings between both methods was found. While WATS3D biopsy may not fully replace traditional biopsies, the two methods have the potential to be combined to increase sensitivity when prognosing $\mathrm{BE}$.

\section{Cytosponge $^{\mathrm{TM}}$}

The Cytosponge ${ }^{\mathrm{TM}}$ is a cell collection device developed to be used in immunohistochemical assays (50). The Cytosponge ${ }^{\mathrm{TM}}$ device is composed of reticulated foam (approximately $30 \mathrm{~mm}$ in diameter) compressed within a gelatin capsule and attached to a string (50). The capsule is then swallowed by the patient, to allow for the dissolution of the gelatin and expansion of the foam, cells are absorbed within the entire length of the esophagus (50). After the cells are retrieved, immunohistochemistry (IHC) is performed to detect expression of markers such as trefoil factor 3 (TFF3). TFF3 is shown to distinguish intestinal cells of
$\mathrm{BE}$ from the other columnar epithelium from the normal upper airway (54). Cell collection by Cytosponge ${ }^{\mathrm{TM}}$ and TFF immunohistochemical analysis is an applicable screening tool, including potentially low cost, suitability for primary care, high tolerability, and diagnostic accuracy (50). Similar to endoscopic brush collection of cytology specimens, cells collected by Cytosponge ${ }^{\mathrm{TM}}$ can be assessed by histological and molecular analyses to simultaneously provide information such as grade of dysplasia and potential for transformation into cancer cells (54).

\section{Molecular diagnostics}

\section{Genomics}

\section{Heritability (genome wide association studies)}

Current efforts are focused on assessing the genome of BE to further understand the disease progression and pathways. One study has attributed $43 \%$ of the GERD phenotype, the precursor to $\mathrm{BE}$, to be heritable, but these numbers are largely unknown in the BE landscape (55). Although previous studies have shown genetic predisposition between $20 \%$ of first- or second-degree relatives with a proposed autosomal-dominant mode of inheritance, it is unlikely that these factors would account for the majority of $\mathrm{BE}$ cases, which are largely sporadic in nature $(55,56)$. Several genome wide association studies (GWAS) have been conducted with respect to BE, most notably by the Wellcome Trust Case Control Consortium and the Barrett's and Esophageal Adenocarcinoma Consortium (57-60). These studies have linked several chromosome regions and loci to BE risk, including the $M H C$ region, FOXF1, FOXP, CRTC1, GDF7, TBX5, ALDH1X2, BARX1 and CTFR $(57-59,61)$. Heritability of this indication has been estimated to be $9.9 \%$, respectively (58). Recently, other approaches, such as genome wide exome analyses have been conducted in hopes of characterizing the genetic variance in BE. A 2018 study investigated the integration of common tracts of homozygosity within exomes and identified signaling pathways significant in BE and EAC such as NOTCH and WNT (62). Importantly, inhibition of NOTCH has been previously found to increase $C D X 2$ gene expression during $\mathrm{BE}$ development. Although more than 20 loci have been found to be significant as of the present, they account for a small minority of the genetic variance in $\mathrm{BE}$, and fail to aid in quantifying the risk of $\mathrm{BE}$ progression to $\mathrm{EAC}(61,63)$. Advancing technologies such as next generation sequencing 
could help in future genome-based investigations, but the current knowledge is not sufficient to quantify and qualify $\mathrm{BE}$ genetic variability.

\section{Gene-environment interactions}

The possibility of genetic elements combined with environmental risk factors has also been studied in terms of $\mathrm{BE}$ risk (64). The largest risk factors that have been associated with BE and EAC are the presence of GERD, obesity and to a lesser degree-smoking status. A 2018 study by Dong et al. investigated these three factors with the aim of identifying associated SNPs in EAC and $\mathrm{BE}$ (62). The study found that BE patients had a higher BMI than control subjects and were more likely to have been previous smokers and report recurrent GERD symptoms (62). BE risk associated with obesity was found to be doubled in persons with rs491603-AA genotype, and significant interactions with smoking exposure were found with chromosome $15 \mathrm{p} 14$, along with several other chromosomes (62). However, none of the gene-environment interactions reached genome-wide significance. A 2019 study found no interactions between smoking, alcohol consumption, or cardiovascular status with the previously found BE genotypes (65). The latter study, however, consisted of a smaller sample size as well as investigated a smaller number of SNPs. The findings of the two studies are, generally, inconclusive and indicate the need for further investigation.

\section{Progression from pre-malignant BE to EC}

There is no current biomarker that is used to selectively determine the risk of a patient with pre-malignant BE to EAC. The current standard of care involves determining the level of dysplasia present in the patient's tissue and stratifying them according to metaplasia, LGD, and HGD (64). However, this technique has clinical limitations due to its dependence on physician observation. For example, there is less than $50 \%$ interobserver agreement when diagnosing tissue with LGD (11). Interpace Diagnostics (Pittsburg, PA) has developed a test based upon mutational load (ML) assessment of the tissue. Trindade et al. found that ML was correlated to the grade of dysplasia, and can aid in histological evaluation of tissue for patients with BE indefinite for dysplasia (66). The test assesses 10 genetic loci of tumor suppressor genes [1p (CMM1, L-myc), 3p (VHL, HoGG1), 5q (MCC, APC), 9p (CDKN2A), 10q (PTEN, MXI1), 17p (TP53), 17q (RNF43, NME1), 18q (SMAD4, DCC), 21q (TFF1, PSEN2) and 22q (NF2)], assigns a value according to the degree of cumulative genetic derangement, and determines risk of progression of $\mathrm{BE}$ indefinite for dysplasia to HGD with $100 \%$ specificity at an ML above 1.5. Another study focused on a methylationbased assay and predicted BE progression to EAC with a sensitivity of up to $50 \%(66,67)$. On another hand, a Northern Ireland group used a biomarker panel with a combination focus on LGD, abnormal DNA ploidy and Aspergillus oryzae lectin and correctly identified $24 \%$ of BE progressors prior to cancer development (68).

\section{Transcriptomics}

\section{RNAseq}

Transcriptomic sequencing has been performed on premalignant and malignant esophageal tissue and used to investigate the noncoding RNA landscape within the disease space. Genes such as BRCA1 and PRKDC have been found as driver genes for EAC in such studies, as well as a 4-gene combination of CTSL, COL17A1, KLF4 and $\mathrm{E} 2 \mathrm{~F} 3$ that helped in providing discrepancy between $\mathrm{BE}$ and EAC tissues (69). Transcriptomic studies have found 685 long noncoding RNAs to be dysregulated in EAC tissue, as well as dysregulation among repeat elements in BE tissue dependent on the stage of the tissue observed (69). Such studies have also been used to identify upregulated transcription factors-EGR1, EGR3, FOSB, FOS, NR4A1, ATF3-in LGD BE and EAC as potential prognostic biomarkers (69). Single cell RNA sequencing techniques have found that $\mathrm{BE}$ cell populations identified by the markers LEFTY1 and OLFM4 display a high amount of transcriptional overlap with esophageal submucosal gland cells, but this overlap is not observed in gastric/duodenal cells (31). The study also found that the markers SPINK4 and ITLN1 serve as a predecessor to goblet cells in $\mathrm{BE}$ which could be promising in identifying metaplasia (31).

\section{Gene expression assays}

Microarray analyses in the $\mathrm{BE}$ disease space have been implemented to identify biomarkers of interest. An analysis from 2009 of three microarray datasets identified DDC and $\mathrm{TFF} 3$ as upregulated in the $\mathrm{BE}$ tissue as compared to normal esophageal tissue (70). While this could serve as a helpful pre-BE identifier, it does not provide information on BE progression thereafter to EAC. Another study focused on expression of microRNA (miRNA) using microarray analysis and found that dysregulation of specific 
miRNAs could serve as a cause of metaplastic processes in the disease $(71,72)$. Interestingly, gene expression studies have found that the stromal and extracellular matrix genes linked to EAC tumor growth are expressed in tissue prior to dysplasia, pointing to the importance of detecting these changes via markers in precancerous BE tissue, as this would provide earlier detection as opposed to real-time physical visual inspection of malignant specimens (73).

\section{Additional diagnostics and proteomics}

\section{Aneuploidy}

In non-dysplastic and LGD patients, aneuploidy due to P16 allelic loss has been observed, specifically in up to $75 \%$ of metaplastic cases (74). Aneuploidy has been found to be present in $90 \%$ of HGD and EAC cases (75). Flow cytometry is used in the current diagnostic landscape to assess the aneuploid characteristics of non-dysplastic and LGD patients to predict their risk of progression (76).

\section{P53}

P53 staining of LGD tissue can be helpful in defining risk of HGD and EAC development along with inspection of the loss of heterozygosity (LOH) of the gene (77). LOH in the tumor suppressor P53 has been observed in both EAC and HGD tissue resulting in a 16 -fold increase in the risk of developing EAC (76). One study found that the mutated gene was found in $72 \%$ of EAC cases and $69 \%$ of LGD cases, but only in $2.5 \%$ of non-dysplastic cases (77). While this could serve as an effective biomarker for EAC progression risk, a conflicting study found that $32.7 \%$ of $\mathrm{BE}$ patients with this type of $P 53 \mathrm{LOH}$ behavior progressed to EAC (78).

\section{Histopathology}

The histopathology of BE is defined by the replacement of the squamous epithelium normally found in the esophagus with columnar epithelium. This morphological change has served as a prognosticator for BE development, and is dependent upon staining of tissue and observer classification. While this change is fairly obvious in initial progression to $\mathrm{BE}$ tissue, distinguishing between LGD and HGD tissue suffers from inter-observer disagreement issues due to the subtlety of changes. Studies have found that there is only $58 \%$ agreement between pathologists when attempting to separate normal esophageal samples from $\mathrm{BE}$ samples, and less than $50 \%$ agreement in diagnosing low grade dysplasia in BE patients $(11,79)$.

\section{IHC/immunofluorescence (IF)—commercial}

IHC has been used in diagnostic, predictive and prognostic BE applications. IHC of TFF3 has been used to screen patients for BE, as well as on P21, P53, and ERCC1 to predict a patient's potential response to chemotherapy (77). IHC analysis of EGFR, Cyclin A, and Cyclin D1 have also been used in a predictive manner (77). Biomarkers for prognostic endpoints have also been investigated using IHC, with markers such as Cyclin D1 and EGFR indicating decreased survival, and COX-2 and VEGF being associated with metastases, recurrence or shorter survival (77). IHC has also been used to identify EPCAM as a potential EAC-specific biomarker, which could be useful as a $\mathrm{BE}$ progression risk marker (80).

Cernostics (Pittsburg, PA) developed a tissue-based BE diagnostic assay $\left(\right.$ TissueCypher $^{\circledR}$ ) which aims to risk stratify LGD patients for EAC. The assay uses IF labeling of K20, p16INK4a, AMACR, p53, HER2/neu, CD68, COX-2, HIF- $1 \alpha$ and CD45RO and subsequent analysis using the TissueCypher ${ }^{\circledR}$ Image Analysis Platform (81). The platform segments cell-based objects and tissue structures such as epithelial and stromal compartments in order to capture morphological information, as well as biomarker intensity from IF labeling (81). In a recent study, the assay sensitivity was $68 \%$ as compared to $76 \%$ by trained pathologists, with assay specificity of $79 \%$ as compared to $64-77 \%$ by pathologists (82). Importantly, the assay detected 50-56\% of $\mathrm{BE}$ disease progressors that had previously been down staged to non-dysplastic BE by pathologists (82). The assay, therefore, demonstrates potential in correctly identifying $\mathrm{BE}$ progression markers that pathologists may not be inspecting during routine screening protocols for this indication.

\section{Mass spectroscopy}

Mass spectrometry has recently been used to investigate and identify BE relevant biomarkers (83). An early study used matrix-assisted laser desorption/ionization mass spectrometry to attempt to identify protein peak differences, but the technology was not compatible with formalinfixed, paraffin-embedded tissue, serving as a problem for general use as the majority of patients are surveilled through biopsies in which the tissue is fixed as such, and an effective mass spectrometry approach would aim to make use of this tissue. Mass spectrometry techniques have recently become compatible with formalin-fixed, paraffin- 
embedded tissue, making the technology potentially useful for BE prognostication (84). The SWATH-MS platform was used to identify proteomic expression trends related to EAC treatment resistance, in particular, cisplatin, 5-FU and taxanes (HMGB1, IL-1RA, LGALS3BP, PRMT1, S100A8, SFN, and TXN) (15). The use of mass spectrometry is novel within the $\mathrm{BE}$ diagnosis landscape but proves promising due to the high-throughput nature of the technology itself using original biopsy tissue and having the ability to use antibody free detection and quantification of multiple biomarkers simultaneously (83).

\section{Imaging for early detection of Barrett's carcinogenesis}

\section{Narrow-band imaging endoscopy (NBI)}

NBI is an optical image enhanced technology that enhances blue and green light to highlight abnormal neoplastic vasculature (85). There are retrospective studies that have evaluated the role of positron emission tomographycomputed tomography (PET-CT) scan in comparison with NBI endoscopy, to detect second primary cancers of head and neck cancer (86). However, this imaging modality showed that NBI endoscopy has high sensitivity for detecting head and neck cancers but the specificity was greatly lower compared to PET/CT scans (86).

\section{Magnified NBI}

Magnified NBI is an endoscopy performed with a magnifying endoscope using a standard video-endoscopic system and narrow-band imaging system (87). However, this imaging modality does not always produce clear imaging or precise gross inspection of diseased tissue due to the background mucosa interfering with the full visibility (88).

\section{Acid sprayed endoscopy}

Acetic-acid spray is a diagnostic method used to observe the in-vivo histology of EAC. This method presents verified histology that shows the opening of the cancerous glands that allows for change in the mucosa color to be white allowing gross inspection of the enhanced surface structure of Barrett's mucosa (89). It is important to note that this diagnostic imaging modality does not provide molecular information about the tissue of interest, thus a conclusion can only be made by the eye of the observer.

\section{$C T$}

Computed tomography (CT) can be implemented in order to stage esophageal tumors, for example, revealing softtissue masses within the esophagus upon transition from BE metaplasia to EAC (90). However, the imaging modality is not able to provide extremely accurate or reliable information (90).

\section{PET-CT}

PET-CT has been shown to have no benefit in patients diagnosed with dysplastic BE but could be beneficial in detecting EAC disease progression, invasion and metastases to regional or distant lymph nodes or organs (44).

\section{Endoscopic ultrasound (EUS)}

EUS is used for diagnosing and staging esophageal cancers, as well as choosing effective treatment options (91). It has been cited as the best diagnostic tool for finding he depth of tumor invasion as well as local lymph node spread, but has also been shown to over- and under-stage patients $(44,91)$. This is also seen when used in BE applications, with depth invasion findings leading to over-staging in the majority of patients (92).

It is important to note that the aforementioned imaging modalities provide no molecular information about the tissue of interest, limiting the clinical utility of disease progression predictability, categorization of dysplasia and if malignant transformations are occurring.

\section{Therapy to prevent or eradicate EAC}

\section{Ablation techniques}

For BE patients with high grade dysplasia, one of the early treatments options is endoscopic ablation. There are different types of ablation techniques used when treating $\mathrm{BE}$. One technique is endoscopic cryotherapy. It is the process of using a rapid cooling agent, such as liquid nitrogen, to induce targeted tissue destruction. Results from a study with $80 \mathrm{BE}$ patients with HGD and LGD treated with two to three cryoablation cycles showed, $91 \%$ complete eradication of dysplasia (CE-D) in LGD patients and $81 \%$ in HGD patients (93). Showing that cryotherapy can be a safe effective method in treating and managing the progression of $\mathrm{BE}$. Another technique is radiofrequency 
ablation (RFA) which is a modality that utilizes heat energy to destroy diseased tissue. RFA has shown $90.5 \%$ CE-D in LGD and $81 \%$ CE-D in HGD patients, compared to $22.7 \%$ in LGD and $19.0 \%$ in HGD in the control group (94). However, once Barrett's tissue has been removed via ablation, patients still require follow-up surveillance to monitor disease recurrence as patients can still progress to cancer within a year of ablative extirpation (95).

\section{Anti-reflux surgical modalities}

Anti-reflux surgery serves as one treatment option for GERD-related symptoms in patients with BE (96). This endoscopic approach facilitates fundoplication, reinforcing the sphincter and relieving inflammation (97). Anti-reflux surgery has shown to provide physiologic and symptomatic control in the maintenance of chronic GERD (98). However, anti-reflux surgery has shown conflicting results in patients with BE. Some studies show that this procedure not only treats GERD-related symptoms, but can also disrupt BE progression and prevent the development of EAC $(99,100)$. Meanwhile, other studies and metaanalyses indicate that only a small percentage of BE patients undergo recovery, and that long-term results of anti-reflux surgery are not as promising as short-term results $(98,101)$. Additionally, BE patients must still undergo routine surveillance following anti-reflux surgery (98). Ultimately, it is inconclusive if anti-reflux surgery reduces the likelihood of carcinogenic development and progression, and thus serves as a therapy for symptom control in BE, while not yet serving as a viable cancer-prevention therapy (96).

\section{Perioperative chemotherapy}

Perioperative chemotherapy methods are used to increase survival rates in early stage EAC patients. The MAGIC trials included the use of epirubicin, cisplatin, and 5-FU (ECF). Perioperative treatment with ECF showed a five-year survival rate of $36 \%$ compared to $23 \%$ for surgery alone (102). Another study known as the ACCORD trials used cisplatin and 5-FU. It saw a five-year survival of $38 \%$ compared to $24 \%$ for surgery alone (102). The FLOT protocol includes the use of 5-FU, leucovorin, oxaliplatin, and docetaxel in preoperative chemotherapy. Perioperative FLOT treatment showed a 58.7\% 3-year survival rate, compared to the $30.9 \%$ for patients that received an esophagectomy first (103). When compared to contemporary neoadjuvant chemoradiation and perioperative chemotherapy, FLOT has become the favorable choice (104). While perioperative chemotherapy treatments have shown to increase overall survival for EAC patients, there is still much work to be done in the field to find the most durable regimen.

\section{Chemoradiation}

Although chemotherapy may show to be beneficial in other forms of cancers; it is not highly effective in treating advanced EAC. Chemotherapy treatments alone vs. no therapy in advance EAC patients did not demonstrate any survival benefits (105). Specific biomarkers associated with EAC that possibly contribute resistance to chemotherapy have been identified. This network of biomarkers associated with increased resistance to taxanes, platins, and anthracyclines were overexpressed or downregulated when observed before the course of chemotherapy treatment for EAC (15). Another treatment option for EAC has been conducting surgical extirpation of the lesion and noncancerous margins. An esophagectomy carries a number of possible complications. This includes leakage, stricture, delayed emptying, chylothorax, and reflux (106). Without additional therapy, it also has a rate of local reoccurrence as high as $35 \%$ (107). The current standard of care is combing therapies in hopes of increasing survival for patients. One of these methods is neoadjuvant chemoradiotherapy (CRT). This combination can downstage the tumor and improve results after surgery. Survivability for patients that underwent this method was 43.2 months, compared to the 27.1 months with surgery alone (108). When looking at adjuvant settings of CRT, the overall 3-year survival went up from $41 \%$ to $50 \%$ and median survival went from 27 months to 36 months compared to surgery alone (109). While this does increase survivability, it does not affect the 5-year mortality rate for the disease. Abdo et al. proposes that molecular diagnostics of proteins associated with increased resistance or enhanced chemosensitivity for all FDA-approved therapies for EAC should be routinely scrutinized to optimize first line therapy for these patients (110).

\section{Targeted therapy}

Up to $90 \%$ of esophageal cancers express EGFR, but antiEGFR therapy does not show to improve survival (111). Because of this, targeted therapy is limited in its use. Currently there are two FDA approved targeted therapy 
treatments for EAC (111). The first one targets HER2. The overexpression of HER2 causes heterodimerization which then promotes oncogenesis (112). This has led to the use of trastuzumab (Herceptin ${ }^{\mathrm{TM}}$ ) as first- or second-line treatment for HER2 positive cases (111). Trastuzumab works by disrupting the HER2 signaling pathway by receptor depletion (113). This treatment has shown to improve survival for patients expressing HER2 at high levels. It is important to note that HER2 positive cases only account for $20 \%$ of esophageal cancers (111). Meaning that for the larger number of EAC patients, this treatment is not an option. The second FDA approved treatment targets VEGF. VEGF was observed as a mediator for angiogenesis in this disease. Ramucirumab is used as a second-line treatment in inhibiting the VEGF pathway. A double blinded, randomized, trial showed that ramucirumab did have a survival benefit over the control (114). It is important to remember these treatments offer a short survival benefit over traditional methods. Further research is required to discover stronger druggable targets for inhibition of disease drivers in EAC lesions.

\section{Immunotherapy}

Immunotherapy helps improve the immune system in recognition and elimination of cancer cells. Recent clinical success in this method includes chimeric antigen t-cell (CAR T) therapy, programmed death-1 (PD-1), and cytotoxic T lymphocyte antigen-4 (CTLA-4) antibody blocking (115). This type of treatment is beneficial because it can be applied to patient populations that may not be eligible for certain targeted therapies. Pembrolizumab (Keytruda ${ }^{\mathrm{TM}}$ ) is a PD1 inhibitor that has been FDA approved for second-line treatment in esophageal cancer (111). Patients that have received prior treatment were observed to have an $11.6 \%$ objective response rate (ORR) after being treated with pembrolizumab (116). To increase the response rate, studies are being conducted using pembrolizumab in conjunction with different treatments. A phase II study using pembrolizumab in combination with cisplatin and fluorouracil showed an ORR of $60 \%$ (117). Ongoing studies for first line treatment are being conducted to analyze the benefits of the combination pembrolizumab with other therapies (117). Another immune checkpoint inhibitor that is being studied is Nivolumab which targets the PD-1/PD-L1 pathway (118). Phase II trials using nivolumab in conjunction with ipilimumab, a monoclonal CTLA-4 targeting antibody, showed an objective response rate of $24 \%(118,119)$. As of 2020 nivolumab was FDA approved for use in patients showing metastatic esophageal squamous cell carcinoma (ESCC).

CheckMate 577 trial was conducted to evaluate nivolumab as a therapy option in resected esophageal or GEJ cancer patients. Results showed that participants who received nivolumab had a median diseases free survival of 22.4 month, compared to the 11.0 month of the placebo group (120). Following this trial, in May 2021 the FDA approved nivolumab for treatment use in patients with complete resection of esophageal or GEJ cancer that have had neoadjuvant chemoradiation therapy.

Esophageal cancers are usually grouped together during studies, but it is important to recognize there is differences among them. A molecular comparison between EAC, ESCC, and GAC revealed there were evident differences between adenocarcinomas and squamous cell carcinoma. ESCC had significantly lower mutational rates compared to EAC and GAC (121). These results raise the question in the validity of grouping these cancers together. Instead, it enforces the theory that treatments should be decided based on histological subtypes and molecular target status.

\section{Conclusions}

EAC is the deadliest and fastest rising cancer in the United States (Figures 1 and 2). With BE as the only known precursor for EAC, advancements in the monitoring of $\mathrm{BE}$ are essential to enable early diagnosis and improve patient outcomes. Although many clinical limitations persist, significant progress has been made in the diagnosis, prognosis, and therapy for Barrett's-related esophageal cancer. EAC's high resistance to chemotherapy has presented a need for the development of effective targeted therapies. Ongoing research of interventions of the EGFR and VEGF pathways, alongside ongoing findings in checkpoint inhibitor trials, show potential for improvement in the clinical treatment of Barrett's-related esophageal cancer. To optimally translate therapeutic developments into clinical utility, progress must be made in the diagnosis of Barrett'srelated esophageal cancer to enable earlier detection and intervention (1). An ability to observe molecular expression patterns that are hallmarks of carcinogenesis would provide gastroenterologists and oncologists insight into the disease's stability.

Advancements in tissue retrieval techniques have paved a road for the adoption of less invasive and more descriptive monitoring, surveillance, and characterization of $\mathrm{BE}$ 
pathogenesis. Significant progress has been made regarding genomic, transcriptomic, and proteomic approaches for molecular diagnostics of Barrett's-related esophageal cancer; however, these research innovations and findings are yet to be translated into full clinical adoption. The current standard diagnostic paradigm suffers from a lack of interobserver agreement and descriptive diagnostic tools to inform the effective and efficient use of therapeutic options. Further research into preventative therapies and the increased use of molecular diagnostics can provide actionable information for clinicians to mitigate the threats presented by Barrett's-related esophageal cancer.

\section{Acknowledgments}

The authors would like to recognize Dr. Sheeno Thyparambil for lending his knowledge on precision diagnostics, Dr. Ajay Bansal for imparting his clinical expertise, thoughts and insight into the upstream patient management strategies for BE-related EAC, and Dr. Christopher Hartley and Dr. Catherine Hagen for advising on the histopathological clinical protocols for these indications. We would also like to thank Professor Dean Lewis and Dr. Ivica Labuda from the Georgetown University Biochemistry Department for their support.

Funding: None.

\section{Footnote}

Reporting Checklist: The authors have completed the Narrative Review reporting checklist. Available at https:// dx.doi.org/10.21037/jgo-21-117

Peer Review File: Available at https://dx.doi.org/10.21037/ jgo-21-117

Conflicts of Interest: All authors have completed the ICMJE uniform disclosure form (available at https:// dx.doi.org/10.21037/jgo-21-117). Dr. Mittal is a clinical consultant for Stella Diagnostics. Dr. Abdo is an employee of a molecular diagnostics company in the GI space, Stella Diagnostics, Inc.. No assays or product candidates developed by Stella Diagnostics are discussed in this manuscript. Dr. Agrawal is funded by the NIH for his research outside of the purview of this manuscript. The work presented in this article was not supported by any NIH grants issued to Dr. Agrawal and his department. The other authors have no conflicts of interest to declare.
Ethical Statement: The authors are accountable for all aspects of the work in ensuring that questions related to the accuracy or integrity of any part of the work were appropriately investigated and resolved.

Open Access Statement: This is an Open Access article distributed in accordance with the Creative Commons Attribution-NonCommercial-NoDerivs 4.0 International License (CC BY-NC-ND 4.0), which permits the noncommercial replication and distribution of the article with the strict proviso that no changes or edits are made and the original work is properly cited (including links to both the formal publication through the relevant DOI and the license). See: https://creativecommons.org/licenses/by-nc-nd/4.0/.

\section{References}

1. Abdo J, Agrawal DK, Mittal SK. Basis for molecular diagnostics and immunotherapy for esophageal cancer. Expert Rev Anticancer Ther 2017;17:33-45.

2. Shahbaz Sarwar CM, Luketich JD, Landreneau RJ, et al. Esophageal cancer: an update. Int J Surg 2010;8:417-22.

3. Kambhampati S, Xu Y, Meltzer S. Risk Factors for Progression of Barrett's Esophagus to High-Grade Dysplasia and Esophageal Carcinoma: 456. Am J Gastroenterol 2016;111:S205-6.

4. Amadi C, Gatenby P. Barrett's oesophagus: Current controversies. World J Gastroenterol 2017;23:5051-67.

5. ASGE Standards of Practice Committee; Muthusamy VR, Lightdale JR, et al. The role of endoscopy in the management of GERD. Gastrointest Endosc 2015;81:1305-10.

6. Siegel R, Naishadham D, Jemal A. Cancer statistics, 2012. CA Cancer J Clin 2012;62:10-29.

7. ASGE Standards of Practice Committee; Evans JA, Early DS, et al. The role of endoscopy in Barrett's esophagus and other premalignant conditions of the esophagus. Gastrointest Endosc 2012;76:1087-94.

8. Kapoor H, Agrawal DK, Mittal SK. Barrett's esophagus: recent insights into pathogenesis and cellular ontogeny. Transl Res 2015;166:28-40.

9. Kamboj AK, Katzka DA, Iyer PG. Endoscopic Screening for Barrett's Esophagus and Esophageal Adenocarcinoma: Rationale, Candidates, and Challenges. Gastrointest Endosc Clin N Am 2021;31:27-41.

10. Choi SE, Hur C. Screening and surveillance for Barrett's esophagus: current issues and future directions. Curr Opin Gastroenterol 2012;28:377-81. 
11. Runge TM, Abrams JA, Shaheen NJ. Epidemiology of Barrett's Esophagus and Esophageal Adenocarcinoma. Gastroenterol Clin North Am 2015;44:203-31.

12. Rajagopal PS, Nipp RD, Selvaggi KJ. Chemotherapy for advanced cancers. Ann Palliat Med 2014;3:203-28.

13. Thompson SK, Sullivan TR, Davies R, et al. Her-2/neu gene amplification in esophageal adenocarcinoma and its influence on survival. Ann Surg Oncol 2011;18:2010-7.

14. Bang YJ, Van Cutsem E, Feyereislova A, et al. Trastuzumab in combination with chemotherapy versus chemotherapy alone for treatment of HER2-positive advanced gastric or gastro-oesophageal junction cancer (ToGA): a phase 3, open-label, randomised controlled trial. Lancet 2010;376:687-97.

15. Mittal S, Abdo J, Agrawal D. Discovery proteomics detects expression trends associated with resistance to the most commonly used chemotherapies in esophageal adenocarcinoma. Eur J Cancer 2020;138:S40.

16. Yang YM, Hong $\mathrm{P}, \mathrm{Xu} W W$, et al. Advances in targeted therapy for esophageal cancer. Signal Transduct Target Ther 2020;5:229.

17. Moinova HR, LaFramboise T, Lutterbaugh JD, et al. Identifying DNA methylation biomarkers for nonendoscopic detection of Barrett's esophagus. Sci Transl Med 2018;10:eaao5848.

18. Patti MG. An Evidence-Based Approach to the Treatment of Gastroesophageal Reflux Disease. JAMA Surg 2016;151:73-8.

19. Sharma P. Clinical practice. Barrett's esophagus. N Engl J Med 2009;361:2548-56.

20. Krishnamoorthi R, Mohan BP, Jayaraj M, et al. Risk of progression in Barrett's esophagus indefinite for dysplasia: a systematic review and meta-analysis. Gastrointest Endosc 2020;91:3-10.e3.

21. Singh S, Garg SK, Singh PP, et al. Acid-suppressive medications and risk of oesophageal adenocarcinoma in patients with Barrett's oesophagus: a systematic review and meta-analysis. Gut 2014;63:1229-37.

22. de Jonge PJ, van Blankenstein M, Looman CW, et al. Risk of malignant progression in patients with Barrett's oesophagus: a Dutch nationwide cohort study. Gut 2010;59:1030-6.

23. Bhat S, Coleman HG, Yousef F, et al. Risk of malignant progression in Barrett's esophagus patients: results from a large population-based study. J Natl Cancer Inst 2011;103:1049-57.

24. Frei NF, Stachler MD, Bergman JJGHM. Risk stratification in Barrett's esophagus patients with diagnoses of indefinite for dysplasia: the definite silver bullet has not (yet) been found. Gastrointest Endosc 2020;91:11-3.

25. Krishnamoorthi R, Borah B, Heien H, et al. Rates and predictors of progression to esophageal carcinoma in a large population-based Barrett's esophagus cohort. Gastrointest Endosc 2016;84:40-46.e7.

26. Grewal US, Randhawa MS, Mehta A. Role of Mitochondrial Markers in Improved Detection and RiskStratification in Barrett's Esophagus Patients. Yale J Biol Med 2019;92:533-9.

27. Porter RJ, Murray GI, Brice DP, et al. Novel biomarkers for risk stratification of Barrett's oesophagus associated neoplastic progression-epithelial HMGB1 expression and stromal lymphocytic phenotype. Br J Cancer 2020;122:545-54.

28. Grin A, Streutker CJ. Histopathology in barrett esophagus and barrett esophagus-related dysplasia. Clin Endosc 2014;47:31-9.

29. Lavery DL, Nicholson AM, Poulsom R, et al. The stem cell organisation, and the proliferative and gene expression profile of Barrett's epithelium, replicates pyloric-type gastric glands. Gut 2014;63:1854-63.

30. Jiang M, Li H, Zhang Y, et al. Transitional basal cells at the squamous-columnar junction generate Barrett's oesophagus. Nature 2017;550:529-33.

31. Owen RP, White MJ, Severson DT, et al. Single cell RNAseq reveals profound transcriptional similarity between Barrett's oesophagus and oesophageal submucosal glands. Nat Commun 2018;9:4261.

32. Que J, Garman KS, Souza RF, et al. Pathogenesis and Cells of Origin of Barrett's Esophagus. Gastroenterology 2019;157:349-364.e1.

33. Lind A, Siersema PD, Kusters JG, et al. The immune cell composition in Barrett's metaplastic tissue resembles that in normal duodenal tissue. PLoS One 2012;7:e33899.

34. Lind A, Siersema PD, Kusters JG, et al. The Microenvironment in Barrett's Esophagus Tissue Is Characterized by High FOXP3 and RALDH2 Levels. Front Immunol 2018;9:1375.

35. Karstens KF, Kempski J, Giannou AD, et al. Antiinflammatory microenvironment of esophageal adenocarcinomas negatively impacts survival. Cancer Immunol Immunother 2020;69:1043-56.

36. Yang L, Lu X, Nossa CW, et al. Inflammation and intestinal metaplasia of the distal esophagus are associated with alterations in the microbiome. Gastroenterology 2009;137:588-97.

37. Snider EJ, Compres G, Freedberg DE, et al. Alterations to 
the Esophageal Microbiome Associated with Progression from Barrett's Esophagus to Esophageal Adenocarcinoma. Cancer Epidemiol Biomarkers Prev 2019;28:1687-93.

38. Lopetuso LR, Severgnini M, Pecere S, et al. Esophageal microbiome signature in patients with Barrett's esophagus and esophageal adenocarcinoma. PLoS One 2020;15:e0231789.

39. Peixoto A, Silva M, Pereira P, et al. Biopsies in Gastrointestinal Endoscopy: When and How. GE Port J Gastroenterol 2016;23:19-27.

40. Pophali P, Halland M. Barrett's oesophagus: diagnosis and management. BMJ 2016;353:i2373.

41. Everson MA, Ragunath K, Bhandari P, et al. How to Perform a High-Quality Examination in Patients With Barrett's Esophagus. Gastroenterology 2018;154:1222-6.

42. Booth CL, Thompson KS. Barrett's esophagus: A review of diagnostic criteria, clinical surveillance practices and new developments. J Gastrointest Oncol 2012;3:232-42.

43. Pohl H, Pech O, Arash H, et al. Length of Barrett's oesophagus and cancer risk: implications from a large sample of patients with early oesophageal adenocarcinoma. Gut 2016;65:196-201.

44. Shaheen NJ, Falk GW, Iyer PG, et al. ACG Clinical Guideline: Diagnosis and Management of Barrett's Esophagus. Am J Gastroenterol 2016;111:30-50; quiz 51.

45. Ghorbian S, Ardekani AM. Non-Invasive Detection of Esophageal Cancer using Genetic Changes in Circulating Cell-Free DNA. Avicenna J Med Biotechnol 2012;4:3-13.

46. Banki F, Mason RJ, Oh D, et al. Plasma DNA as a molecular marker for completeness of resection and recurrent disease in patients with esophageal cancer. Arch Surg 2007;142:533-8; discussion 538-9.

47. Tomita H, Ichikawa D, Ikoma D, et al. Quantification of circulating plasma DNA fragments as tumor markers in patients with esophageal cancer. Anticancer Res 2007;27:2737-41.

48. Kern E, Lin D, Larson A, et al. Prospective assessment of the diagnostic utility of esophageal brushings in adults with eosinophilic esophagitis. Dis Esophagus 2016;29:48-53.

49. Technology Assessment Committe; Barkun A, Liu J, et al. Update on endoscopic tissue sampling devices. Gastrointest Endosc 2006;63:741-5.

50. di Pietro M, Chan D, Fitzgerald RC, et al. Screening for Barrett's Esophagus. Gastroenterology 2015;148:912-23.

51. Pilot Study of Esocheck in Eosinophilic Esophagitis Full Text View - ClinicalTrials.gov n.d. Available online: https://clinicaltrials.gov/ct2/show/NCT04311970 (accessed December 16, 2020).

52. Pilot Study of EsoCheck Compared to Biopsies and Brush Cytology During Endoscopy for Evaluation of Eosinophilic Esophagitis - Medical Research n.d. Available online: https://clinicalresearch.itmat.upenn.edu/ clinicaltrial/6302/medical-research-pilot-study-esocheckcompared/ (accessed December 16, 2020).

53. Smith M, Clayton S. WATS 3D Brush Biopsy Variability Compared to Traditional Forceps Biopsy in the Identification of Barrett's Esophagus and Dysplasia Diagnosis: 324. Am J Gastroenterol 2017;112:S177.

54. Sanghi V, Thota PN. Barrett's esophagus: novel strategies for screening and surveillance. Ther Adv Chronic Dis 2019;10:2040622319837851.

55. Findlay JM, Middleton MR, Tomlinson I. Genetic susceptibility to Barrett's oesophagus: Lessons from early studies. United European Gastroenterol J 2016;4:485-92.

56. Drovdlic CM, Goddard KA, Chak A, et al. Demographic and phenotypic features of 70 families segregating Barrett's oesophagus and oesophageal adenocarcinoma. J Med Genet 2003;40:651-6.

57. Su Z, Gay LJ, Strange A, et al. Common variants at the MHC locus and at chromosome 16q24.1 predispose to Barrett's esophagus. Nat Genet 2012;44:1131-6.

58. Palles C, Chegwidden L, Li X, et al. Polymorphisms near TBX5 and GDF7 are associated with increased risk for Barrett's esophagus. Gastroenterology 2015;148:367-78.

59. Levine DM, Ek WE, Zhang R, et al. A genome-wide association study identifies new susceptibility loci for esophageal adenocarcinoma and Barrett's esophagus. Nat Genet 2013;45:1487-93.

60. Ek WE, Levine DM, D'Amato M, et al. Germline genetic contributions to risk for esophageal adenocarcinoma, Barrett's esophagus, and gastroesophageal reflux. J Natl Cancer Inst 2013;105:1711-8.

61. Gharahkhani P, Fitzgerald RC, Vaughan TL, et al. Genome-wide association studies in oesophageal adenocarcinoma and Barrett's oesophagus: a large-scale meta-analysis. Lancet Oncol 2016;17:1363-73.

62. Dong J, Levine DM, Buas MF, et al. Interactions Between Genetic Variants and Environmental Factors Affect Risk of Esophageal Adenocarcinoma and Barrett's Esophagus. Clin Gastroenterol Hepatol 2018;16:1598-1606.e4.

63. Wanchai V, Jin J, Bircan E, et al. Genome-wide tracts of homozygosity and exome analyses reveal repetitive elements with Barrets esophagus/esophageal adenocarcinoma risk. BMC Bioinformatics 2019;20:98.

64. Kailasam A, Mittal SK, Agrawal DK. Epigenetics in the 
Pathogenesis of Esophageal Adenocarcinoma. Clin Transl Sci 2015;8:394-402.

65. Crous-Bou M, Jovani M, De Vivo I, et al. GeneEnvironment Interactions and the Risk of Barrett's Esophagus in Three US Cohorts. Am J Gastroenterol 2019;114:893-9.

66. Trindade AJ, McKinley MJ, Alshelleh M, et al. Mutational load may predict risk of progression in patients with Barrett's oesophagus and indefinite for dysplasia: a pilot study. BMJ Open Gastroenterol 2019;6:e000268.

67. $\mathrm{Wu} \mathrm{W}$, Bhagat TD, Yang X, et al. Hypomethylation of noncoding DNA regions and overexpression of the long noncoding RNA, AFAP1-AS1, in Barrett's esophagus and esophageal adenocarcinoma. Gastroenterology 2013;144:956-966.e4.

68. Fels Elliott DR, Fitzgerald RC. Molecular markers for Barrett's esophagus and its progression to cancer. Curr Opin Gastroenterol 2013;29:437-45.

69. Maag JLV, Fisher OM, Levert-Mignon A, et al. Novel Aberrations Uncovered in Barrett's Esophagus and Esophageal Adenocarcinoma Using Whole Transcriptome Sequencing. Mol Cancer Res 2017;15:1558-69.

70. Lao-Sirieix P, Boussioutas A, Kadri SR, et al. Nonendoscopic screening biomarkers for Barrett's oesophagus: from microarray analysis to the clinic. Gut 2009;58:1451-9.

71. Wijnhoven BP, Hussey DJ, Watson DI, et al. MicroRNA profiling of Barrett's oesophagus and oesophageal adenocarcinoma. Br J Surg 2010;97:853-61.

72. Gu J, Wang Y, Wu X. MicroRNA in the pathogenesis and prognosis of esophageal cancer. Curr Pharm Des 2013;19:1292-300.

73. Hao Y, Triadafilopoulos G, Sahbaie P, et al. Gene expression profiling reveals stromal genes expressed in common between Barrett's esophagus and adenocarcinoma. Gastroenterology 2006;131:925-33.

74. Jenkins GJ, Doak SH, Parry JM, et al. Genetic pathways involved in the progression of Barrett's metaplasia to adenocarcinoma. Br J Surg 2002;89:824-37.

75. Morales CP, Souza RF, Spechler SJ. Hallmarks of cancer progression in Barrett's oesophagus. Lancet 2002;360:1587-9.

76. Fouad YM, Mostafa I, Yehia R, et al. Biomarkers of Barrett's esophagus. World J Gastrointest Pathophysiol 2014;5:450-6.

77. Maitra I, Date RS, Martin FL. Towards screening Barrett's oesophagus: current guidelines, imaging modalities and future developments. Clin J Gastroenterol
2020;13:635-49.

78. Murray L, Sedo A, Scott M, et al. TP53 and progression from Barrett's metaplasia to oesophageal adenocarcinoma in a UK population cohort. Gut 2006;5 5:1390-7.

79. Sharma P. Low-grade dysplasia in Barrett's esophagus. Gastroenterology 2004;127:1233-8.

80. O'Neill JR, Pak HS, Pairo-Castineira E, et al. Quantitative Shotgun Proteomics Unveils Candidate Novel Esophageal Adenocarcinoma (EAC)-specific Proteins. Mol Cell Proteomics 2017;16:1138-50.

81. Critchley-Thorne RJ, Davison JM, Prichard JW, et al. A Tissue Systems Pathology Test Detects Abnormalities Associated with Prevalent High-Grade Dysplasia and Esophageal Cancer in Barrett's Esophagus. Cancer Epidemiol Biomarkers Prev 2017;26:240-8.

82. Frei NF, Khoshiwal AM, Konte K, et al. Tissue Systems Pathology Test Objectively Risk Stratifies Barrett's Esophagus Patients With Low-Grade Dysplasia. Am J Gastroenterol 2021;116:675-82.

83. Abdo J, Wichman CS, Dietz NE, et al. Discovery of Novel and Clinically Relevant Markers in Formalin-Fixed Paraffin-Embedded Esophageal Cancer Specimen. Front Oncol 2018;8:157.

84. Prieto DA, Hood BL, Darfler MM, et al. Liquid Tissue: proteomic profiling of formalin-fixed tissues. Biotechniques 2005;Suppl:32-5.

85. Boeriu A, Boeriu C, Drasovean S, et al. Narrow-band imaging with magnifying endoscopy for the evaluation of gastrointestinal lesions. World J Gastrointest Endosc 2015;7:110-20.

86. Su HA, Hsiao SW, Hsu YC, et al. Superiority of NBI endoscopy to PET/CT scan in detecting esophageal cancer among head and neck cancer patients: a retrospective cohort analysis. BMC Cancer 2020;20:69.

87. Yoshida T, Inoue H, Usui S, et al. Narrow-band imaging system with magnifying endoscopy for superficial esophageal lesions. Gastrointest Endosc 2004;59:288-95.

88. Kuznetsov K, Lambert R, Rey JF. Narrow-band imaging: potential and limitations. Endoscopy 2006;38:76-81.

89. Yamagata T, Hirasawa D, Fujita N, et al. Efficacy of acetic acid-spraying method in diagnosing extension of Barrett's cancer under the squamous epithelium. Dig Endosc 2012;24:309-14.

90. Iyer R, Dubrow R. Imaging of esophageal cancer. Cancer Imaging 2004;4:125-32.

91. Krill T, Baliss M, Roark R, et al. Accuracy of endoscopic ultrasound in esophageal cancer staging. J Thorac Dis 2019;11:S1602-9. 
92. Fernández-Sordo JO, Konda VJ, Chennat J, et al. Is Endoscopic Ultrasound (EUS) necessary in the pretherapeutic assessment of Barrett's esophagus with early neoplasia? J Gastrointest Oncol 2012;3:314-21.

93. Lal P, Thota PN. Cryotherapy in the management of premalignant and malignant conditions of the esophagus. World J Gastroenterol 2018;24:4862-9.

94. Shaheen NJ. Radiofrequency ablation for barrett esophagus with dysplasia. Gastroenterol Hepatol 2010;6:223-4.

95. Akiyama J, Roorda A, Triadafilopoulos G. Managing Barrett's esophagus with radiofrequency ablation. Gastroenterol Rep (Oxf) 2013;1:95-104.

96. Falk GW, Wani S. 25 - Barrett's Esophagus: Diagnosis, Surveillance, and Medical Management. In: Chandrasekhara V, Elmunzer BJ, Khashab MA, et al. editors. Clinical Gastrointestinal Endoscopy, 3rd ed. Philadelphia: Elsevier, 2019:279-290.e5.

97. Narsule CK, Burch MA, Ebright MI, et al. Endoscopic fundoplication for the treatment of gastroesophageal reflux disease: initial experience. J Thorac Cardiovasc Surg 2012;143:228-34.

98. Morrow E, Bushyhead D, Wassenaar E, et al. The impact of laparoscopic anti-reflux surgery in patients with Barrett's esophagus. Surg Endosc 2014;28:3279-84.

99. Knight BC, Devitt PG, Watson DI, et al. Long-term Efficacy of Laparoscopic Antireflux Surgery on Regression of Barrett's Esophagus Using BRAVO Wireless pH Monitoring: A Prospective Clinical Cohort Study. Ann Surg 2017;266:1000-5.

100. Maret-Ouda J, Konings P, Lagergren J, et al. Antireflux Surgery and Risk of Esophageal Adenocarcinoma: A Systematic Review and Meta-analysis. Ann Surg 2016;263:251-7.

101. Ortega Deballon P. Indications for Anti-Reflux Surgery in Barrett's Esophagus. Cir Esp 2015;93:270-1.

102. Donohoe CL, Reynolds JV. Neoadjuvant treatment of locally advanced esophageal and junctional cancer: the evidence-base, current key questions and clinical trials. J Thorac Dis 2017;9:S697-704.

103. Wang K, Ren Y, Ma Z, et al. Docetaxel, oxaliplatin, leucovorin, and 5-fluorouracil (FLOT) as preoperative and postoperative chemotherapy compared with surgery followed by chemotherapy for patients with locally advanced gastric cancer: a propensity score-based analysis. Cancer Manag Res 2019;11:3009-20.

104.Hoeppner J, Lordick F, Brunner T, et al. ESOPEC: prospective randomized controlled multicenter phase
III trial comparing perioperative chemotherapy (FLOT protocol) to neoadjuvant chemoradiation (CROSS protocol) in patients with adenocarcinoma of the esophagus (NCT02509286). BMC Cancer 2016;16:503.

105. Adenis A, Penel N, Horn S, et al. Palliative chemotherapy does not improve survival in metastatic esophageal cancer. Oncology 2010;79:46-54.

106. Chen KN. Managing complications I: leaks, strictures, emptying, reflux, chylothorax. J Thorac Dis 2014;6 Suppl 3:S355-63.

107. Mukherjee K, Chakravarthy AB, Goff LW, et al. Esophageal adenocarcinoma: treatment modalities in the era of targeted therapy. Dig Dis Sci 2010;55:3304-14.

108. Kwong DLW, Lam KO. Radiotherapy for Esophageal Adenocarcinoma. Esophageal Adenocarcinoma, Springer, 2018:7-17.

109. Jabbour SK, Thomas CR Jr. Radiation therapy in the postoperative management of esophageal cancer. J Gastrointest Oncol 2010;1:102-11.

110.Abdo J, Agrawal DK, Mittal SK. "Targeted" Chemotherapy for Esophageal Cancer. Front Oncol 2017;7:63.

111. Barsouk A, Rawla P, Hadjinicolaou AV, et al. Targeted Therapies and Immunotherapies in the Treatment of Esophageal Cancers. Med Sci (Basel) 2019;7:100.

112. Connell CM, Doherty GJ. Activating HER2 mutations as emerging targets in multiple solid cancers. ESMO Open 2017;2:e000279.

113. Gerson JN, Skariah S, Denlinger CS, et al. Perspectives of HER2-targeting in gastric and esophageal cancer. Expert Opin Investig Drugs 2017;26:531-40.

114. Fontana E, Sclafani F, Cunningham D. Anti-angiogenic therapies for advanced esophago-gastric cancer. Indian J Med Paediatr Oncol 2014;35:253-62.

115. Yang Y. Cancer immunotherapy: harnessing the immune system to battle cancer. J Clin Invest 2015;125:3335-7.

116. Fuchs CS, Doi T, Jang RW, et al. Safety and Efficacy of Pembrolizumab Monotherapy in Patients With Previously Treated Advanced Gastric and Gastroesophageal Junction Cancer: Phase 2 Clinical KEYNOTE-059 Trial. JAMA Oncol 2018;4:e180013.

117.Joshi SS, Maron SB, Catenacci DV. Pembrolizumab for treatment of advanced gastric and gastroesophageal junction adenocarcinoma. Future Oncol 2018;14:417-30.

118.Zayac A, Almhanna K. Esophageal, gastric cancer and immunotherapy: small steps in the right direction? Transl Gastroenterol Hepatol 2020;5:9.

119. Yamamoto S, Kato K. Immuno-oncology for esophageal 
cancer. Future Oncol 2020;16:2673-81.

120. Kelly RJ, Ajani JA, Kuzdzal J, et al. Adjuvant Nivolumab in Resected Esophageal or Gastroesophageal Junction Cancer. N Engl J Med 2021;384:1191-203.

Cite this article as: Mittal SK, Abdo J, Adrien MP, Bayu BA, Kline JR, Sullivan MM, Agrawal DK. Current state of prognostication, therapy and prospective innovations for Barrett's-related esophageal adenocarcinoma: a literature review. J Gastrointest Oncol 2021;12(4):1197-1214. doi: 10.21037/ jgo-21-117
121. Salem ME, Puccini A, Xiu J, et al. Comparative Molecular Analyses of Esophageal Squamous Cell Carcinoma, Esophageal Adenocarcinoma, and Gastric Adenocarcinoma. Oncologist 2018;23:1319-27. 